\title{
An Analytical Approach to Integral Resonant Control of Second-Order Systems
}

\author{
Mohammad Namavar, Student Member, IEEE, Andrew J. Fleming, Member, IEEE, Majid Aleyaasin, \\ K. Nakkeeran, Member, IEEE, and Sumeet S. Aphale, Member, IEEE
}

\begin{abstract}
Systems with colocated sensor-actuator pairs exhibit the interesting property of pole-zero interlacing. Integral resonance control (IRC) exploits this property by changing the polezero interlacing to zero-pole interlacing. The unique phase response of this class of systems enables a simple integral feedback controller to add substantial damping. Over the past few years, IRC has proven to be extremely versatile and has been applied to a wide variety of systems whose dominating dynamics of interest can be accurately modeled by second-order transfer functions. To date, a manual approach has been employed to determine the parameters of the IRC scheme, namely the feed-through term and the integral gain. In this paper, the relationship between the feed-through term, integral gain, and achievable damping is derived analytically for undamped/lightly damped second-order systems. The relationship between damping controller and an outer servo loop is also derived. These results add to the current understanding of colocated systems and automate the design of IRC controllers with a specified damping and tracking bandwidth. The presented results are applied to design and implement a damping and tracking controller for a piezoelectric nanopositioning stage.
\end{abstract}

Index Terms-Integral resonance control (IRC), nanopositioning, vibration damping.

\section{INTRODUCTION}

$\mathbf{U}$ NWANTED excitation of system resonances can produce vibration that can substantially degrade the performance and life time of many mechatronic systems [1]. Both passive and active damping techniques have been widely reported in the literature. Passive damping techniques have the advantage of needing no sensing or supervisory control but can be limited in performance and may be sensitive to changes in system resonance frequency. Active techniques may be more complicated but have the potential to overcome the performance limitations of passive systems [2].

Manuscript received July 18, 2012; revised December 3, 2012 and January 21, 2013; accepted February 21, 2013. Date of publication April 3, 2013; date of current version February 20, 2014. Recommended by Technical Editor K. K. Leang.

M. Namavar, M. Aleyaasin, and K. Nakkeeran are with the School of Engineering, University of Aberdeen, Aberdeen, AB24 3UE, U.K. (e-mail: Mohammad.namavar@abdn.ac.uk; m.Aleyaasin@abdn.ac.uk; K.Nakkeeran@ abdn.ac.uk)

A. J. Fleming is with the School of Electrical Engineering and Computer Science, The University of Newcastle, Callaghan, NSW 2308, Australia (e-mail: andrew.fleming@newcastle.edu.au).

S. S. Aphale is with the Center for Applied Dynamics Research, School of Engineering, University of Aberdeen, Aberdeen, AB24 3UE, U.K. (e-mail: s.aphale@abdn.ac.uk).

Color versions of one or more of the figures in this paper are available online at http://ieeexplore.ieee.org.

Digital Object Identifier 10.1109/TMECH.2013.2253115
Active vibration control is commonly used to damp systems where bandwidth, precision, or life time are key performance requirements, for example, robotic manipulators [3]-[5], diskdrives [6], aircraft wings [7], nanopositioning stages [8], [9], scanning probe microscopes [10], and high-density memory storage devices [11].

A number of well-performing damping controllers such as the integral force feedback (IFF) [1], shunt damping (SD) [12], positive position feedback (PPF) [13], positive velocity and position feedback (PVPF) [14], resonant control [15], and robust control [16] have been proposed earlier. Apart from the IFF technique, a drawback of other controller designs is that they result in high-order controllers for multimode resonant systems. This makes them suitable only for damping systems where generally the first resonance mode is highly dominant over all the others. To overcome this issue, integral resonance control (IRC) was proposed as a simple, low-order scheme capable of damping multiple modes while retaining high-stability margins [17]. It should be noted that due to the choice of user-selected feed-through term, the IRC incorporates more design flexibility than the IFF scheme, which requires additional sensor and instrumentation.

The general concept of IRC design is to change the pole-zero interlacing of a colocated system $G(s)$ to zero-pole interlacing. This is achieved by adding a constant feed-through term $d$ to the system. An integral controller $C(s)=k_{d} / s$ is then applied to the modified system $\hat{G}(s)=G(s)+d$. As the integral gain $k_{d}$ is increased, the poles of the system move away from the imaginary axis into the left-half complex plane and eventually move to the open-loop zero locations.

Due to the intuitive approach, simplicity, and robust performance, IRC has been successfully employed to damp a variety of systems such as cantilever beams [17], flexible robotic manipulators [18], nanopositioning platforms [19], commercial atomic force microscopes [10], flexible civil structures [20], and walking-induced floor vibrations [21]. It was further shown in [22] that the IRC was a negative imaginary system. This result provided further insight into the structure and stability of IRC implementations [23]. However, the IRC design procedure still requires a trial-and-error approach to determine the necessary feed-through term and integrator gain.

High-precision micro and nanopositioning systems have garnered a substantial amount of research interest in recent years [24]. These precision positioners form an integral part of many specialized technological systems (such as atomic force microscopes) where nanopositioning is a key enabling technology [25]. The frequency response of such systems typically 
shows their first resonant mode being dominant over all the subsequent higher frequency modes. Consequently, they have traditionally been modeled as simple second-order systems with a low damping coefficient [26], [27]. This is the motivation for focusing this paper on the analytical treatment of similar second-order systems.

This paper starts by quantifying the impact of the selection of the feed-through term $d$ on the location of the introduced zeros. An analytic expression is derived that relates the feed-through term $d$ and the integral gain $k_{d}$ to the modal damping $\zeta$. As many applications also require a servo control loop the relationship between the damping gain $k_{d}$ and the integral tracking gain $k_{t}$ is also considered. A limit is identified for the product of the two gains beyond which the overall system is unstable. Experimental results on a piezoelectric-stack actuated nanopositioner (modeled as a second-order system with a low damping coefficient) are presented to validate the theory.

\section{FeED-Through FOR A SECOND-ORDER COLOCATED SYSTEM}

Second-order systems with a lightly damped resonance mode and colocated (or approximately colocated) sensors and actuators are commonly seen in many technological systems. Nanopositioning systems are an example where the presence of a lightly damped resonance mode severely limits the performance. Various damping controllers have been proposed to alleviate this problem [9]. Assuming that the system is decoupled, the dynamics of each axis can be approximated as a secondorder system, that is,

$$
G(s)=\frac{\gamma^{2}}{s^{2}+2 \zeta \omega_{p} s+\omega_{p}^{2}}+d_{f}
$$

where $\zeta$ is the damping coefficient, $\omega_{p}$ is the natural frequency, and $d_{f}$ is a feed-through term introduced to improve the prediction of zero-locations of a truncated model, and to approximate the stiffness of the system at high frequencies [28]. A common assumption is that $\zeta$ has a small value and can be neglected during the mathematical analysis.

Theorem 1: If a feed-through term $d$ is added to a resonant second-order system transfer function given by $G(s)=$ $\gamma^{2} /\left(s^{2}+\omega_{p}^{2}\right)$, then the relationship between the feed-through term and the location of the feed-through-induced zeros $z_{1}$ and $z_{2}$ is as follows.

1) If $d<d_{c}$, then the zeros are imaginary and conjugate, $z_{1}, z_{2}= \pm j \omega_{z}$ and $\omega_{z}^{2}<\omega_{p}^{2}$, where $d_{c}=-\gamma^{2} / \omega_{p}^{2}$.

2) If $d>0$, then the zeros are imaginary and conjugate, $z_{1}, z_{2}= \pm j \omega_{z}$ and $\omega_{z}^{2}>\omega_{p}^{2}$.

3) If $d \in\left[d_{c}, 0\right)$, then the zeros are real.

Proof:

$$
\hat{G}(s)=\frac{\gamma^{2}}{s^{2}+\omega_{p}^{2}}+d=\frac{\gamma^{2}+d\left(s^{2}+\omega_{p}^{2}\right)}{s^{2}+\omega_{p}^{2}}=\frac{d\left(s^{2}+\omega_{z}^{2}\right)}{s^{2}+\omega_{p}^{2}} .
$$

Zeros in $\hat{G}(s)$ are introduced by the feed-through term.

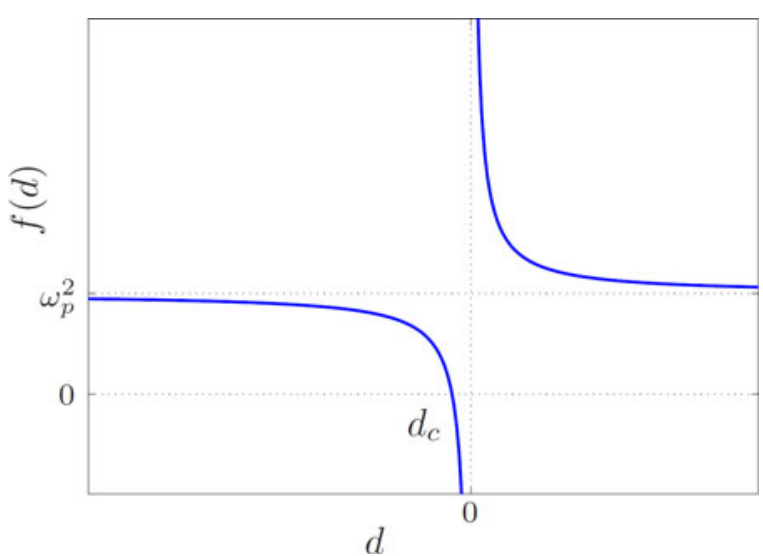

Fig. 1. Plot of $f(d)=\omega_{p}^{2}+\gamma^{2} / d$ versus $d$.

Equation (2) leads to the introduced zeros to be located at

$$
z_{1}, z_{2}= \pm j \sqrt{\left(\omega_{p}^{2}+\frac{\gamma^{2}}{d}\right)}= \pm j \sqrt{f(d)}
$$

When (3) is evaluated in order to find the location of zeros, it shows that the zeros introduced by the feed-through term $d$ are not always imaginary for any arbitrary choice of $d$. Plotting $f(d)$ shows that for a critical value of $d$ given by $d_{c}=-\gamma^{2} / \omega_{p}^{2}$, where $f\left(d_{c}\right)=0$, results in two zeros at the origin. In the range of $\left[d_{c}, 0\right), f(d)$ is negative, resulting in two real zeros (see Fig. 1).

Remark: Note that implementing an IRC scheme on a system manipulated to have zeros as Case 3 will lead to instability since it will be nonminimum phase. Similarly, for $d$ corresponding to Case 2 zeros are introduced at a frequency higher than the system poles, leading to an unstable closed loop since the departure angle for the pole in the origin will be $0^{\circ}$. Consequently, a choice of $d$ corresponding to Case 1 is appropriate for implementing the IRC scheme. This ensures zero-pole interlacing.

Lemma 1: Consider a second-order system $G(s)$ with two purely imaginary poles at $\pm j \omega_{p}$, to which a feed-through term $d$ which satisfies $-\infty<d<d_{c}<0$ is added to introduce a pair of purely imaginary zeros at $\pm j \omega_{z}$. If an integrator, $C(s)=k_{d} / s$, is implemented in positive feedback with $\hat{G}(s)=G(s)+d$, then

1) For all $d \in\left(-\infty, 1.125 d_{c}\right)$ where $\omega_{z}>\omega_{p} / 3$, the rootlocus behaves such that as the integral gain increases, the system pole traverses a curve and reaches the introduced zero without intersecting the real axis. The pole at the origin (introduced by the integrator) gradually goes to $-\infty$ as the integral gain $k_{d}$ tends to $\infty$ [see Fig. 2(a)].

2) When $\omega_{z} \leq \omega_{p} / 3, d \in\left[1.125 d_{c}, d_{c}\right)$, the root-loci starting from the system poles intersect the real axis. In this case the root locus plot could have one [see Fig. 2(b)] or two [see Fig. 2(c)] breakaway points on the negative real axis.

Proof: To find the breakaway points, the characteristic equation should be rearranged such that the gain term $k_{d}$ can be 


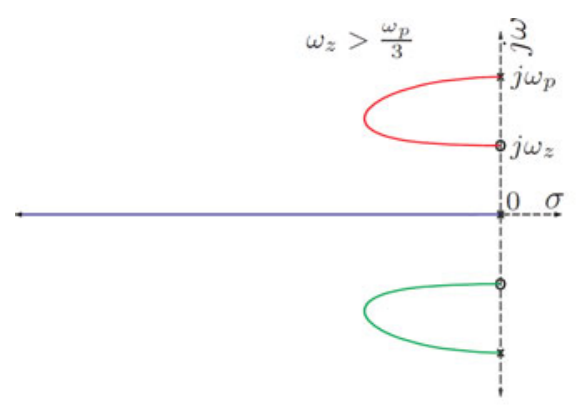

(a)

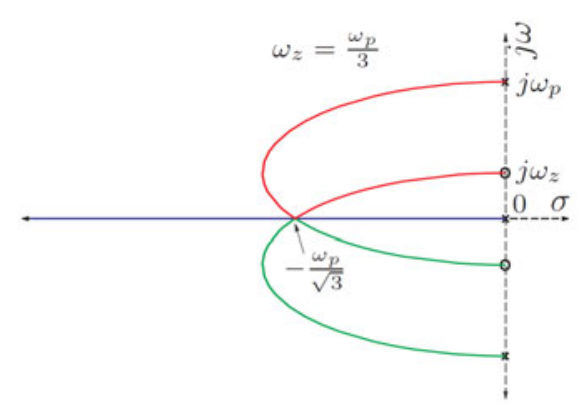

(b)

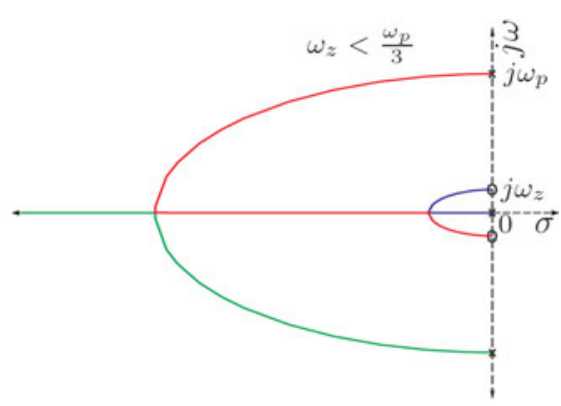

(c)

Fig. 2. In case of $\omega_{z}<\omega_{p}$ root locus of the system with IRC could follow different trajectories. If $\omega_{z} \leq \omega_{p} / 3$, root locus plot will have one (b) or two (c) breakaway points on negative real axis while if $\omega_{z}>\omega_{p} / 3$ root locus has no breakaway points (a) and $\zeta_{\max }<1$.

isolated [29]

$$
\begin{aligned}
& 1-\hat{G}(s) C(s)=0 \\
& k_{d}-\frac{s\left(s^{2}+\omega_{p}^{2}\right)}{d\left(s^{2}+\omega_{z}^{2}\right)}=0 \\
& k_{d}-P(s)=0 .
\end{aligned}
$$

The breakaway points are the roots of $\frac{\mathrm{d}}{\mathrm{d} s} P(s)$. Therefore,

$$
\frac{\mathrm{d}}{\mathrm{d} s} P(s)=\frac{s^{4}+s^{2}\left(3 \omega_{z}^{2}-\omega_{p}^{2}\right)+\omega_{p}^{2} \omega_{z}^{2}}{d\left(s^{2}+\omega_{z}^{2}\right)^{2}}=0 .
$$

Define $x \equiv s^{2}$, reduce the order of equation to two

$$
x^{2}+x\left(3 \omega_{z}^{2}-\omega_{p}^{2}\right)+\omega_{p}^{2} \omega_{z}^{2}=0 .
$$

Since the breakaway points lie on the real axis, conditions that give real roots for (5) need to be found. So

$\left(3 \omega_{z}^{2}-\omega_{p}^{2}\right)^{2}-4 \omega_{p}^{2} \omega_{z}^{2} \geq 0 \Rightarrow \omega_{p}^{2}-5 \omega_{z}^{2} \geq 4 \omega_{z}^{2} \Rightarrow \omega_{p} \geq 3 \omega_{z}$.

For the special case when $\omega_{z}=\omega_{p} / 3$, at a certain controller gain, all three poles converge to the same point on the real axis. This breakaway point occurs at $s=-\omega_{p} / \sqrt{3}$. It can also be shown that the feed-through term is $d=1.125 d_{c}$.

Fig. 2 shows, for $\omega_{z}>\omega_{p} / 3$, the maximum damping $\zeta_{\max }<$ 1 , which results in an underdamped complex conjugate pair where $s=-\zeta \omega_{p} \pm j \omega_{p} \sqrt{1-\zeta^{2}}$.

For $\omega_{z} \in\left(0, \omega_{p} / 3\right]$ which is related to the selection of $d$ in the range $\left[1.125 d_{c}, d_{c}\right]$ results in a pair of imaginary zeros where the root locus intersects with the negative real axis. In this case $\zeta_{\max }>1$ is achievable for some selection of $k_{d}$, and results in an overdamped system.

\section{Relationship BetweEn FeEd-Through, DAMPING, AND IRC GAIN}

In this Section, the relationship between the maximum damping, feed-through term, and controller gain is explored. A similar result for systems that are zero-pole interlaced to begin with has been presented in [30]. Fig. 3, illustrates the root locus behavior for such system.

Theorem 2: Consider a colocated system with a pair of imaginary poles at $\pm j \omega_{p}$ and feed-through-induced imaginary zeros where $\omega_{z}>\omega_{p} / 3$. If the IRC strategy is implemented the max-

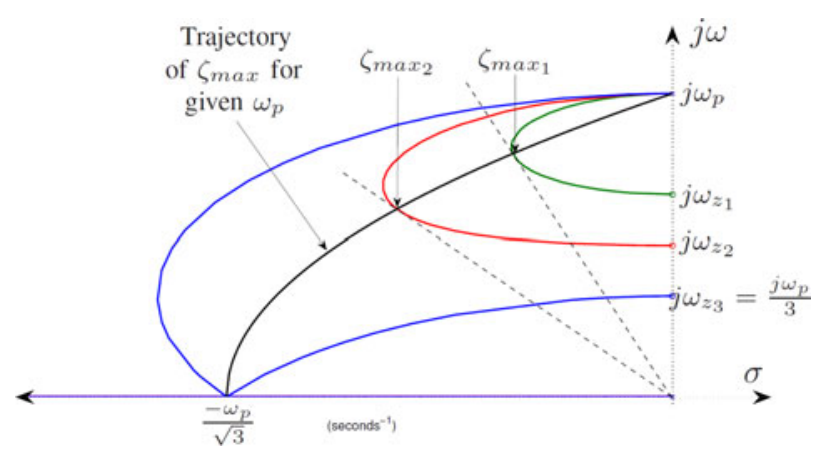

Fig. 3. For $\omega_{z} \in\left(\omega_{p} / 3, \omega_{p}\right)$, the root locus will not have a breakaway point and exhibit a unique point at which $\zeta_{\max }$ as given in (6) is less than 1 . The corresponding integrator gain is given by (7).

imum damping achievable is given by

$$
\zeta_{\max }=\frac{1}{2}\left(\frac{\omega_{p}}{\sqrt{\omega_{p}^{2}+\gamma^{2} / d}}-1\right) .
$$

The controller gain required to reach this maximum damping is given by

$$
k_{d}=\frac{1}{|d|}\left(\omega_{p} \sqrt{\frac{\omega_{p}}{\sqrt{\omega_{p}^{2}+\gamma^{2} / d}}}\right)
$$

where $\omega_{z}=\sqrt{\omega_{p}^{2}+\gamma^{2} / d}$ with respect to feed-through term $d$.

Proof: The closed-loop poles of the system must satisfy

$$
1-\hat{G}(s) C(s)=0
$$

which can be expressed as angle criterion (8) and magnitude criterion (9)

$$
\begin{aligned}
& \angle \hat{G}(s) C(s)=2 k \pi(k \in \mathbb{Z}) \\
& |\hat{G}(s) C(s)|=1
\end{aligned}
$$

where $\hat{G}=\frac{d\left(s^{2}+\omega_{z}^{2}\right)}{s^{2}+\omega_{p}^{2}}$ and $C(s)=\frac{k_{d}}{s}$.

As $s=\sigma+j \omega$,

$$
\arctan \left(\frac{\operatorname{Im}(L(s))}{\operatorname{Re}(L(s))}\right)=2 k \pi
$$

where $L(s)=d\left(s^{2}+\omega_{z}^{2}\right) /\left(s\left(s^{2}+\omega_{p}^{2}\right)\right)$. 
Applying the tan function to both sides of (10) and rearranging results in

$\omega^{4}-\omega^{2}\left(\omega_{z}^{2}+\omega_{p}^{2}-2 \sigma^{2}\right)+\sigma^{4}+\sigma^{2}\left(3 \omega_{z}^{2}-\omega_{p}^{2}\right)+\omega_{p}^{2} \omega_{z}^{2}=0$.

Equation (11) is the equation for the root locus, where closedloop complex-valued poles traverse as $k_{d}$ changes from 0 to $+\infty$. The equation of a line passing through the origin is

$$
\omega=-m \sigma \text {. }
$$

Substituting $\omega$ from (12) into (11) results in an equation based on the unknowns $\sigma$ and $m$. Rearranging in the ascending order of $\sigma$,

$$
\begin{aligned}
\left(m^{4}+2 m^{2}+1\right) \sigma^{4}+\left(-m^{2}\left(\omega_{z}^{2}+\omega_{p}^{2}\right) 3 \omega_{z}^{2}\right. & \left.-\omega_{p}^{2}\right) \sigma^{2} \\
& +\omega_{z}^{2} \omega_{p}^{2}=0 .
\end{aligned}
$$

At the point where maximum damping occurs, the line has one unique point-of-contact with the root-locus. For this point, the discriminant of (13) should be zero and therefore it will have two identical roots. Thus,

$$
\begin{aligned}
\left(-m^{2}\left(\omega_{z}^{2}+\omega_{p}^{2}\right)+\left(3 \omega_{z}^{2}-\omega_{p}^{2}\right)\right)^{2} & \\
& -4\left(m^{4}+2 m^{2}+1\right)\left(\omega_{z}^{2} \omega_{p}^{2}\right)=0 .
\end{aligned}
$$

Expanding and rearranging (14) for ascending order of $m$ gives

$$
\begin{aligned}
\left(\omega_{z}^{2}-\omega_{p}^{2}\right)^{2} m^{4}-2 m^{2}\left(3 \omega_{z}^{4}-\omega_{p}^{4}\right. & \left.+6 \omega_{z}^{2} \omega_{p}^{2}\right) \\
& +9 \omega_{z}^{4}+\omega_{p}^{2}-10 \omega_{z}^{2} \omega_{p}^{2}=0 .
\end{aligned}
$$

Solving the aforementioned expression for $m^{2}$ gives

$$
\begin{aligned}
m^{2} & =\frac{3 \omega_{z}^{4}-\omega_{p}^{4}+6 \omega_{z}^{2} \omega_{p}^{2} \pm 8 \omega_{z}^{3} \omega_{p}}{\left(\omega_{p}^{2}-\omega_{z}^{2}\right)^{2}} \\
m^{2}+1 & =\frac{4 \omega_{z}^{2}\left(\omega_{z} \pm \omega_{p}\right)^{2}}{\left(\omega_{p}-\omega_{z}\right)^{2}\left(\omega_{p}+\omega_{z}\right)^{2}} .
\end{aligned}
$$

Note that the damping factor $\zeta$ of any complex pole $s=\sigma+j \omega$ based on the slope of the line passing through the origin is

$$
\begin{aligned}
\sigma & =-\zeta \omega_{n} \\
\omega & =\omega_{n} \sqrt{1-\zeta^{2}} \\
\zeta & =\frac{1}{\sqrt{m^{2}+1}}
\end{aligned}
$$

where $\omega_{n}=\sqrt{\sigma^{2}+\omega^{2}}$.

Positive and negative sign in (15) can be analyzed separately considering damping factor $\zeta$ should be positive and less than one for an underdamped system.

Case 1: Positive sign in (15), $\left(\omega_{z}+\omega_{p}\right)^{2}$ :

$$
m^{2}+1=\frac{4 \omega_{z}^{2}\left(\omega_{z}+\omega_{p}\right)^{2}}{\left(\omega_{p}-\omega_{z}\right)^{2}\left(\omega_{p}+\omega_{z}\right)^{2}}=\frac{4 \omega_{z}^{2}}{\left(\omega_{p}-\omega_{z}\right)^{2}} .
$$

Substituting the aforementioned expression into (16) yields

$$
\zeta=\frac{\omega_{p}-\omega_{z}}{2 \omega_{z}} .
$$

Since $0<\omega_{p}<3 \omega_{z}, 0<\omega_{p}-\omega_{z}<2 \omega_{z}$ thus,

$$
0<\frac{\omega_{p}-\omega_{z}}{2 \omega_{z}}<1 \Rightarrow \ll \zeta<1
$$

This value for $\zeta$ is valid.

Case 2: Negative sign in (15), $\left(\omega_{z}-\omega_{p}\right)^{2}$. Following the same procedure as Case 1 results in

$$
\zeta=\frac{\omega_{p}+\omega_{z}}{2 \omega_{z}}
$$

Simplifying (18) gives $\zeta>1$ which contradicts with $0<\zeta<1$.

As the derivations are based on having only one point-ofcontact between the locus and the line, this damping factor is the maximum value achievable for a certain selection of $\omega_{p}$ and $\omega_{z}$ and is given by (17). To find out the gain where maximum damping occurs, the magnitude criterion (9) is used. Real and imaginary coordinates of the point where maximum damping occurs are found using (12), (13), and (15). This gives

$$
\left(\left.\sigma\right|_{\zeta_{\max }}\right)^{2}=\frac{\omega_{p}\left(\omega_{p}-\omega_{z}\right)^{2}}{4 \omega_{z}}
$$

and

$$
\left(\left.\omega\right|_{\zeta_{\max }}\right)^{2}=\frac{-\omega_{p}\left(\omega_{p}+\omega_{z}\right)\left(\omega_{p}-3 \omega_{z}\right)}{4 \omega_{z}} .
$$

Substituting (19) and (20) into (9), will result in an expression for integrator gain at maximum damping $\left.k_{d}\right|_{\zeta_{\text {max }}}$ :

$$
\begin{gathered}
\left|\frac{\left(\sigma_{\max }+j \omega_{\max }\right)^{2}+\omega_{z}^{2}}{\left(\sigma_{\max }+j \omega_{\max }\right)\left(\left(\sigma_{\max }+j \omega_{\max }\right)^{2}+\omega_{p}^{2}\right)}\right|=\left|\frac{1}{\left.k_{d}\right|_{\zeta_{\max }}}\right| \\
\left.k_{d}\right|_{\zeta_{\max }}=\frac{1}{d}\left(\omega_{p} \sqrt{\frac{\omega_{p}}{\omega_{z}}}\right) .
\end{gathered}
$$

Note that (19) could theoretically result in $\sigma>0$. As this would imply unstable pole locations, this case is omitted from the analysis.

\section{A. Trajectory of $\zeta_{\max }$}

Proposition 1: Consider a colocated system with poles at $\pm j \omega_{p}$. For all feed-through-term induced zero combinations where $\omega_{p}>\omega_{z}>\omega_{p} / 3$, the locus of possible pole locations corresponding to maximum damping for a specific value of $\omega_{p}$ is determined by

$$
\omega^{2}=\sigma^{2} \pm 2 \sigma \sqrt{\sigma^{2}+\omega_{p}^{2}}+\omega_{p}^{2} .
$$

Proof: To find an expression in terms of the real value $\sigma$, the imaginary value $\omega$, and $\omega_{p}$, one can start by putting (12) in the form

$$
\frac{\omega^{2}}{\sigma^{2}}=m^{2}=\frac{1-\zeta_{\max }^{2}}{\zeta_{\max }^{2}}
$$




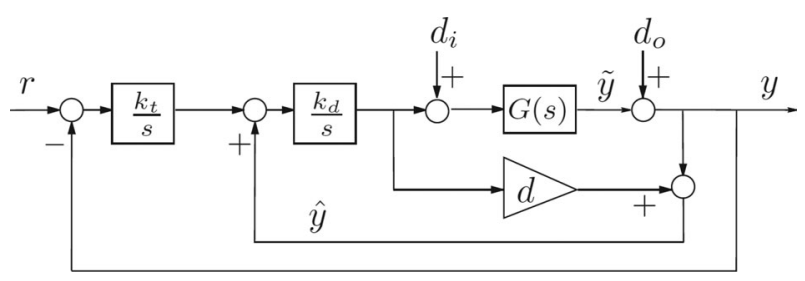

Fig. 4. Block diagram for the IRC damping controller in addition to integral tracking controller scheme where $d$ is the feed-through term, $k_{d}$ is the IRC damping gain and $k_{t}$ is the integral tracking gain.

by using the expression in (16). Using (17), the aforementioned expression can be put in the form

$$
\frac{\omega^{2}}{\sigma^{2}}=\frac{\omega_{z} \omega_{p}-\sigma^{2}}{\sigma^{2}}
$$

The expression in (19) can be rearranged as

$$
\begin{aligned}
\omega_{p}\left(\omega_{p}-\omega_{z}\right)^{2} & =4 \sigma^{2} \omega_{z} \\
\omega_{p} \omega_{z}^{2}-2\left(\omega_{p}^{2}+2 \sigma^{2}\right) \omega_{z}+\omega_{p}^{3} & =0
\end{aligned}
$$

which can be solved for $\omega_{z}$, which yields

$$
\omega_{z}=\frac{\omega_{p}^{2}+2 \sigma^{2} \pm \sqrt{\left(\omega_{p}^{2}+2 \sigma^{2}\right)^{2}-\omega_{p}^{4}}}{\omega_{p}}
$$

Substituting (24) into (23) yields the expression in (22).

The expression in (22) describes the location of the poles that yield the maximum achievable damping coefficient $\zeta$ for a specific $\omega_{p}$ when varying the feed-through term $d$, and can be used to find value for the feed-through term $d$ that maximizes damping.

\section{TRACKInG CONTROLleR}

The IRC algorithm has been applied to damp the resonances of various precision positioning systems, especially nanopositioners [10], [18]-[21]. Nanopositioning system generally employs piezoelectric actuators that tend to introduce nonlinear effects such as hysteresis and creep. To minimize the positioning errors introduced by these phenomena, a damping controller such as IRC, PPF, PVPF, and resonant control is used in conjunction with a simple integral tracking scheme [9], [10], [31]. A block diagram of the typical control scheme incorporating both IRC damping and integral tracking is shown in Fig. 4.

In earlier experiments, it was observed that the tracking and damping controller gains could not be arbitrarily tuned independent of each other. In several experiments, it was seen that increasing the tracking gain beyond a certain limit reduced the damping of the system. In Fig. 4, the transfer functions of interest for performance are $y(s) / r(s)$ and $y(s) / d_{i}(s)$. The transfer function $\tilde{y}(s) / d_{o}(s)$ is also of interest when considering the sensitivity to sensor noise. The characteristic equation for all of the transfer functions mentioned earlier is given by the numerator of

$$
\begin{aligned}
1- & \left(-\frac{k_{t} k_{d} \gamma^{2}}{s^{2}\left(s^{2}+\omega_{p}^{2}\right)}+\frac{k_{d}\left(d\left(s^{2}+\omega_{p}^{2}\right)+\gamma^{2}\right)}{s\left(s^{2}+\omega_{p}^{2}\right)}\right) \\
& =1-\frac{-k_{t} k_{d} \gamma^{2}+k_{d} d s^{3}+k_{d}\left(d \omega_{p}^{2}+\gamma^{2}\right) s}{s^{2}\left(s^{2}+\omega_{p}^{2}\right)} \\
& =\frac{s^{4}-k_{d} d s^{3}+\omega_{p}^{2} s^{2}-k_{d}\left(d \omega_{p}^{2}+\gamma^{2}\right) s+k_{t} k_{d} \gamma^{2}}{s^{2}\left(s^{2}+\omega_{p}^{2}\right)} .
\end{aligned}
$$

Proposition 2: Let $k_{d}$ and $k_{t}$ be the IRC damping and integral tracking gains, respectively. For a closed-loop system as implemented in Fig. 4 to be stable, the gains must obey the following inequality:

$$
k_{t} k_{d}<-\frac{\gamma^{2}+d \omega_{p}^{2}}{d^{2}} .
$$

Proof: To check the stability of the closed-loop transfer function of the system given in Fig. 4, the zeros of (25) should be evaluated. The system is stable if all the zeros have negative real parts. All necessary and sufficient conditions for stability are met if 1) all the coefficients of (25) are positive and 2) all elements of the first column of the Routh-Hurwitz table are positive. As $k_{t}, k_{d}, \gamma$, and $\omega_{p}$ are all positive and $d<d_{c}<0$, the condition for stability can be given by

$$
d \omega_{p}^{2}+\gamma^{2}+k_{t} k_{d} d^{2}<0
$$

Rearranging (27) for $k_{t} k_{d}$ results in the expression (26).

This theorem formally proves the earlier finding that damping and tracking gains are related in the IRC scheme and cannot be tuned independent of each other. This theorem shows that if one gain is increased, the limit for the other is reduced and also gives a limit for the two gains beyond which the overall closedloop system will become unstable. It must be noted that due to several structural similarities between the IRC, IFF, and resistive SD techniques, the stability criteria proved earlier will hold for the IFF and SD control designs (with minor modifications).

Lemma 2: For a given second-order system controlled using the scheme shown in Fig. 4, there exists an absolute maximum value for $k_{t} k_{d}$. The corresponding maximum value is related to $d$ by

$$
\begin{aligned}
d & =-2 \frac{\gamma^{2}}{\omega_{p}^{2}}=2 d_{c} \\
\max \left\{k_{t} k_{d}\right\} & =\frac{\omega_{p}^{4}}{4 \gamma^{2}} .
\end{aligned}
$$

Proof: Define $g(d)=-\left(\gamma^{2}+d \omega_{p}^{2}\right) / d^{2}$. The maximum of $g(d)$ is the absolute maximum of achievable value for product of $k_{t}$ and $k_{d}$. The corresponding value of feed-through term $d$ which maximizes this product can be computed by equating the first derivative of $g(d)$ with respect to $d$ to zero

$$
\begin{gathered}
\frac{\mathrm{d}}{\mathrm{d} d} g(d)=\frac{d \omega_{p}^{2}+2 \gamma^{2}}{d^{3}}=0 \\
\Rightarrow d=-2 \frac{\gamma^{2}}{\omega_{p}^{2}}=2 d_{c} .
\end{gathered}
$$




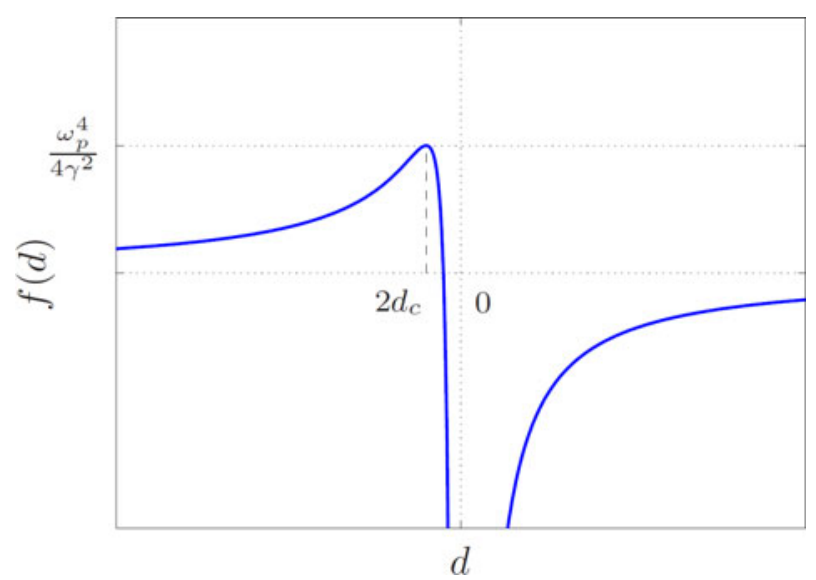

Fig. 5. $g(d)=-\left(\gamma^{2}+d \omega_{p}^{2}\right) / d^{2}$ is the upper limit for $k_{t} k_{d}$. Maximum of this function occurs at $d=2 d_{c}$ and the corresponding value for this product is $\omega_{p}^{4} /\left(4 \gamma^{2}\right)$. Selection of $d>d_{c}$ is not valid choice as $k_{t} k_{d}$ becomes negative which contradicts the precondition $k_{t}>0$ and $k_{d}>0$.

The maximum value for $k_{t} k_{d}$ is given by

$$
\max \left\{k_{t} k_{d}\right\}=\left.g(d)\right|_{d=-2 d_{c}}=\frac{\omega_{p}^{4}}{4 \gamma^{2}} .
$$

Fig. 5 shows this relationship graphically.

\section{EXPERIMENTS}

In this section, the control scheme depicted in Fig. 4 is implemented on a nanopositioning stage.

\section{A. Experimental Setup}

The performance of each controller will be evaluated on a two-axis serial kinematic nanopositioner, pictured in Fig. 6(a). The nanopositioner was designed and constructed at the EasyLab, University of Nevada, Reno, USA. The stage is driven by two $10 \mathrm{~mm} 200-\mathrm{V}$ piezoelectric stack actuators which provide a range of $40 \mu \mathrm{m}$ in each axis. The position is measured by a Microsense 6810 capacitive sensor and 6504-01 probe with a sensitivity of $0.4 \mathrm{~V} / \mu \mathrm{m}$. The stage is driven by two PiezoDrive PDL200 voltage amplifiers with a gain of $20 \mathrm{~V} / \mathrm{V}$.

A second-order model of the system was procured by frequency domain least squares fit. The frequency response of the $x$-axis is compared to the model response in Fig. 6(b). The model parameters are as follows:

$$
G(s)=\frac{2.025 \times 10^{7}}{s^{2}+48.63 s+1.042 \times 10^{7}} .
$$

\section{B. Results}

Using Lemma 2, a feed-through term $d=-3.88$ was deemed suitable as it introduced a zero at $363 \mathrm{~Hz}$ (below the natural frequency of $514 \mathrm{~Hz}$ ). Also, the maximum product of tracking and damping gains that resulted in a stable closed-loop system was $k_{t} k<1.341268 \times 10^{6}$. As seen from Fig. 7, the combination of the two gains given by a generic point $\left(k_{t}, k_{d}\right)$ must lie in the region below the solid red line to ensure stability. Furthermore, to maintain the magnitude of the damped peak of

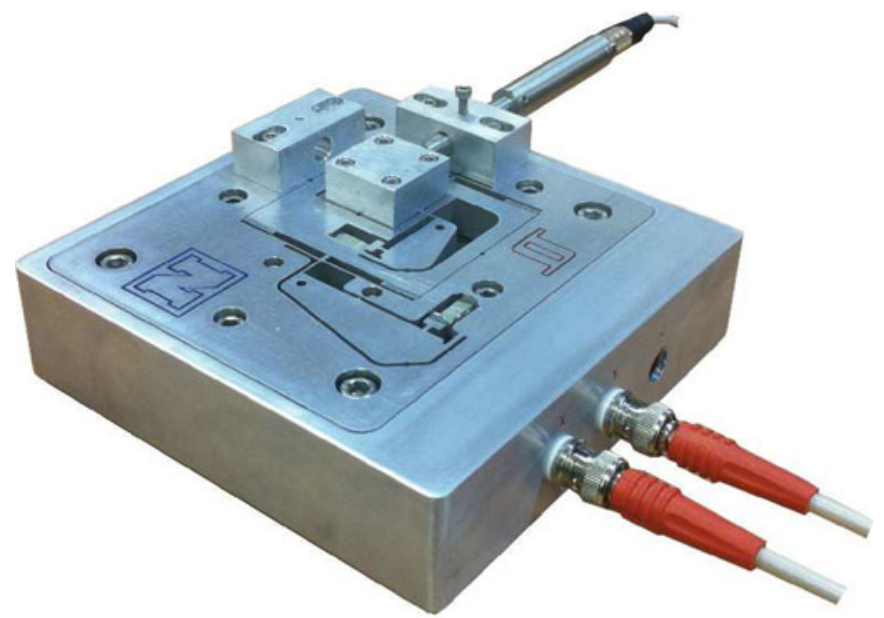

(a)
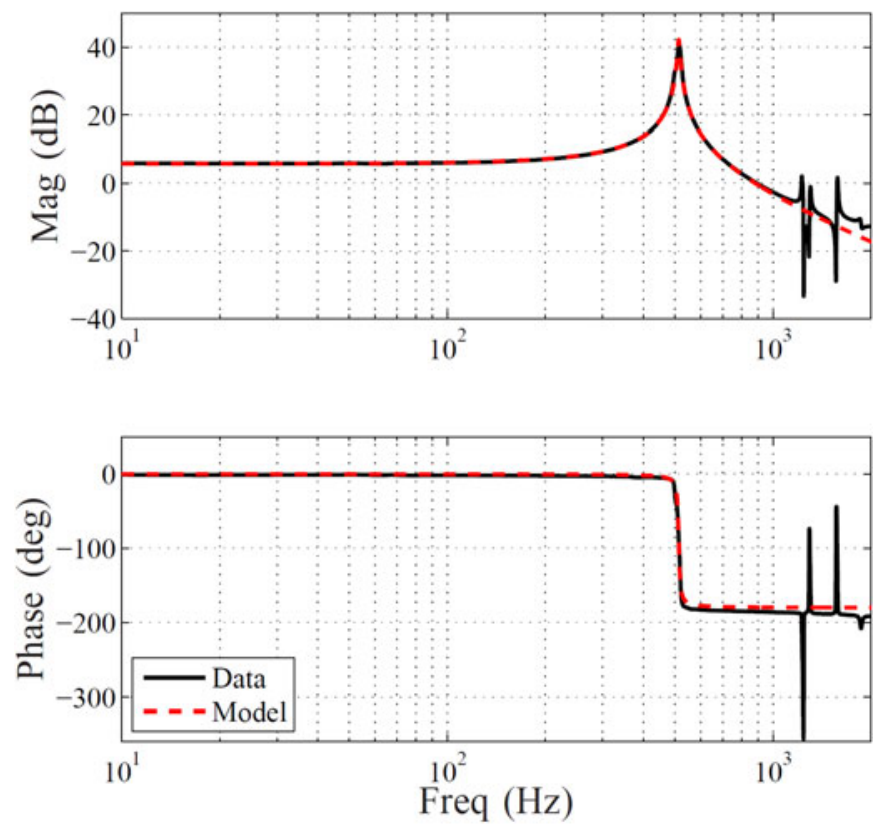

(b)

Fig. 6. (a) Two-axis $40 \mu \mathrm{m}$ serial kinematic nanopositioner designed at the EasyLab, University of Nevada, Reno, NV, USA. (b) Open-loop frequency response of the nanopositioner measured from the voltage amplifier input to the sensor output, scaled to $\mu \mathrm{m} / \mathrm{V}$.

the overall closed-loop (damped + tracking) to be less than the damped system (with no tracking), the choice of selectable gain combinations must be restricted to points below the solid black line. Note that the normalized bandwidth plotted in Fig. 7 is computed by dividing the bandwidth values obtained for each $k_{d}-k_{t}$ combination (within the chosen range) by the resulting overall maximum bandwidth. A similar strategy is utilized to normalize the maximum peak values. Three gain combinations were selected and experimentally implemented. The three cases experimentally tested are as follows.

1) Case 1: Gain corresponding to best achievable damping $k_{d}=987$ and remaining gain (from $k_{t} k_{d}$ ) for tracking, $k_{t}=1357$. It can be clearly seen from Fig. 7 that this point lies beyond the minimum resonance magnitude of 


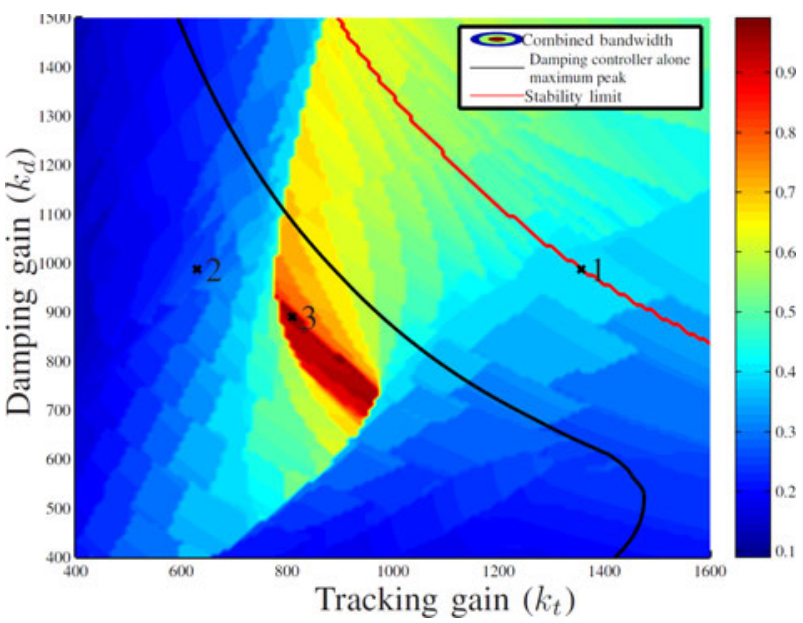

Fig. 7. Evolution of three parameters with respect to tracking gain $k_{t}$ and damping gain $k_{d}$. The first parameter is plotted as a solid contour of the overall normalized bandwidth. The second parameter plotted as the solid black line is the maximum normalized peak of the damped system with no tracking controller. The third parameter plotted as the solid red line is the evolution of the stability limit criteria $k_{t} k_{d}<1.341268 \times 10^{6}$.
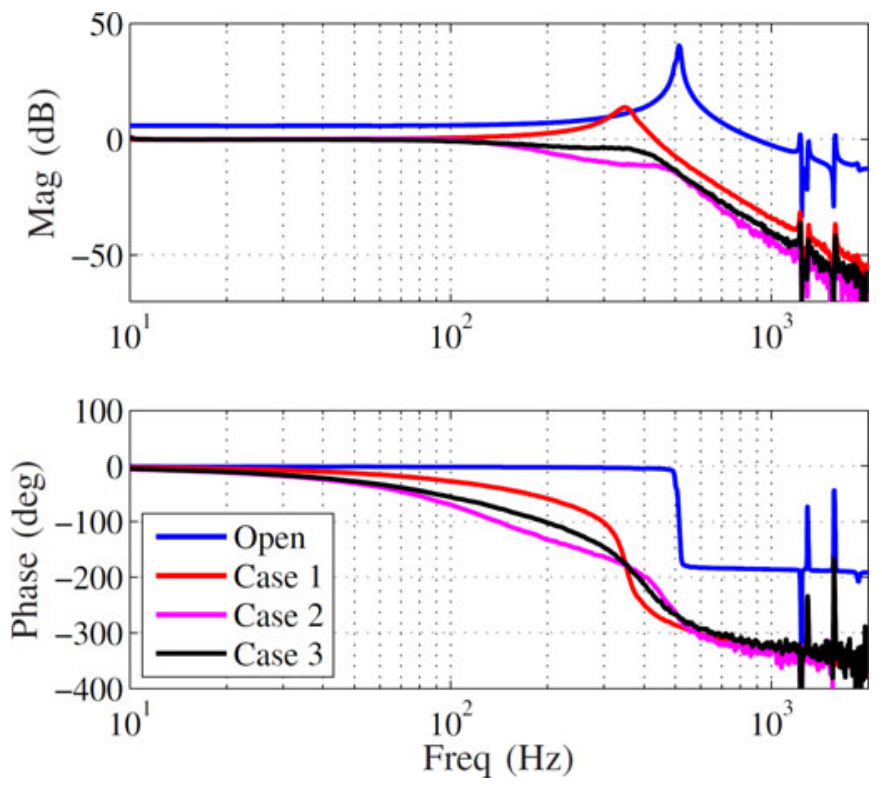

Fig. 8. Open- and closed-loop frequency responses of the system, measured from the reference input to the displacement in micrometer.

the damped system contour (solid black line). This results in some high-frequency ripples being manifested in the time domain plots shown in Fig. 9.

2) Case 2: Gain corresponding to best achievable damping $k_{d}=987$ and tracking gain of $k_{t}=630$ (found via simulations) which results in maximum scan range achievable with respect to $\pm 1 \%$ error allowed. As clearly seen from Fig. 7 this point lies within the minimum resonant magnitude of damped system contour. As a result almost no high frequency ripples are visible in the time domain plots Fig. 9.

3) Case 3: A suitable gain distribution that results in maximum overall bandwidth is extracted from the contour plot

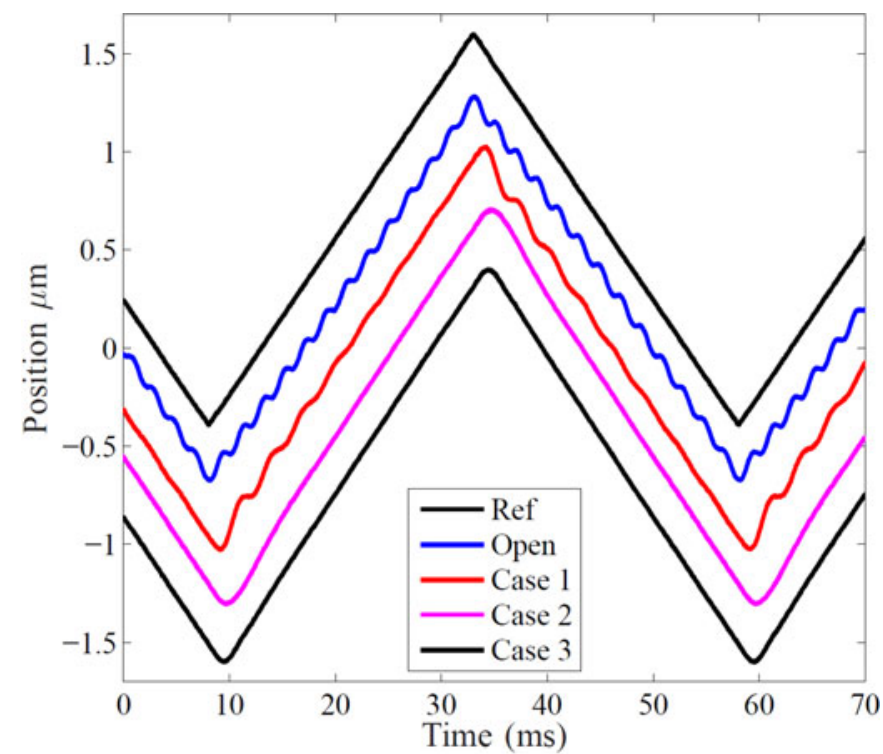

(a)

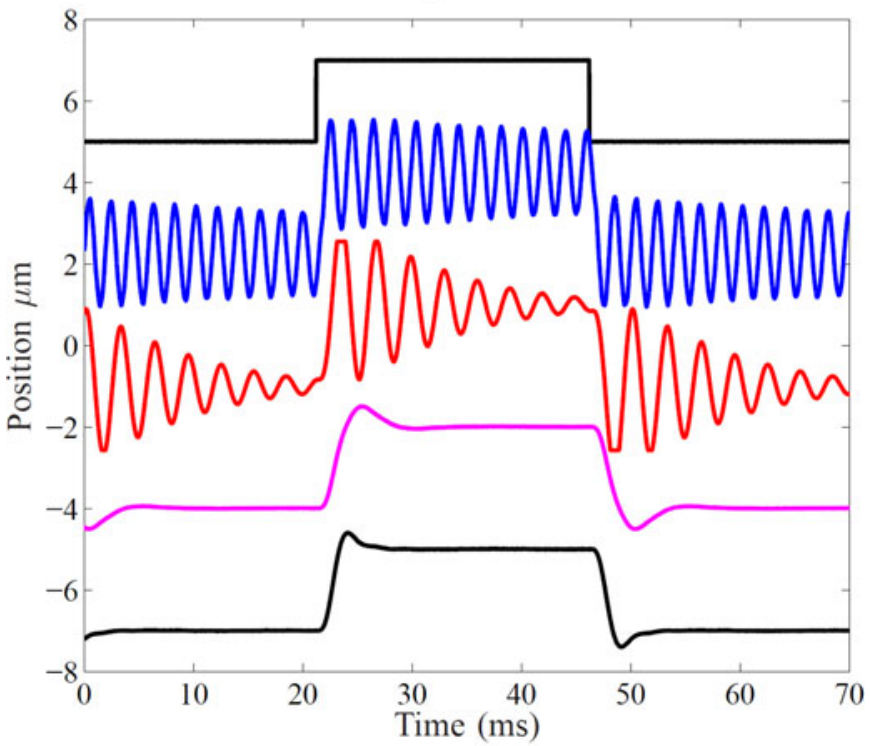

(b)

Fig. 9. (a) Closed-loop response to a $20-\mathrm{Hz} 2-\mu \mathrm{m}$ triangular reference signal. For clarity, the waveforms are offset from each other by $0.3 \mu \mathrm{m}$. (b) Closed-loop response to a $20-\mathrm{Hz} 2-\mu \mathrm{m}$ step change in the reference signal. For clarity, the waveforms are offset from each other by $3 \mu \mathrm{m}$.

given in Fig. 7 (any point in the dark red region): $k_{d}=890$ and $k_{t}=810$.

For this particular application (nanopositioning), maximizing the typical $-3 \mathrm{~dB}$ bandwidth would result in substantial positioning errors (insufficiently damped system resonance could potentially amplify the higher frequency input components). Therefore, a more restrictive bandwidth criteria has been applied and is defined as a range of frequencies where the closed-loop magnitude response of the overall system lies between $\pm 1 \mathrm{~dB}$. The closed-loop frequency responses for Case 1, Case 2, and Case 3 are plotted in Fig. 8. The time-domain responses to a triangular and square-wave reference input are plotted in Fig. 9. In Fig. 8, it can be observed that Case 1 results in a substantial 
damping but is still capable of amplifying higher frequency components of the input triangle wave. Selecting gains as prescribed in Case 2 clearly improves the damping but the tracking bandwidth is reduced. Selection of gains as specified in Case 3 results in the maximum positioning bandwidth of $400 \mathrm{~Hz}$. The system responds well to both triangle wave and square wave inputs.

\section{CONCLUSION}

In this paper, analytical expressions were derived that relate the parameters of an IRC to the closed-loop pole locations, for second-order systems. As many practical applications also require a servo controller, the relationship between the damping and tracking controller gains ( $k_{d}$ and $\left.k_{t}\right)$ and the closed-loop stability was also found. These relationships were experimentally verified on a nanopositioning stage. The relationships derived in this paper form a basis for further optimization of the popular IRC damping scheme and will lead to the development of improved control strategies that combine damping as well as tracking.

\section{REFERENCES}

[1] A. Preumont, Vibration Control of Active Structures: An Introduction. Dordrecht, The Netherlands: Kluwer, 1997.

[2] D. J. Inman, Vibration With Control, Measurement, and Stability. Englewood Cliffs, NJ, USA: Prentice-Hall, 1989.

[3] T. N. Chang, R. Kwadzogah, and R. J. Caudill, "Vibration control of linear robots using a piezoelectric actuator," IEEE/ASME Trans. Mechatronics, vol. 8, no. 4, pp. 439-445, Dec. 2003.

[4] J. Park, P. H. Chang, H. S. Park, and E. Lee, "Design of learning input shaping technique for residual vibration suppression in an industrial robot," IEEE/ASME Trans. Mechatronics, vol. 11, no. 1, pp. 55-65, Feb. 2006.

[5] S. C. P. Gomes, V. S. da Rosa, and B. de Carvalho Albertini, "Active control to flexible manipulators," IEEE/ASME Trans. Mechatronics, vol. 11, no. 1, pp. 75-83, Feb. 2006

[6] H. Numasato and M. Tomizuka, "Settling control and performance of a dual-actuator system for hard disk drives," IEEE/ASME Trans. Mechatronics, vol. 8, no. 4, pp. 431-438, Dec. 2003.

[7] P. P. Friedmann and T. A. Millott, "Vibration reduction in rotorcraft using active control: A comparison of various approaches," J. Guidance Contr. Dyn., vol. 18, no. 4, pp. 664-673, 1995.

[8] S. Verma, W.-J. Kim, and J. Gu, "Six-axis nanopositioning device with precision magnetic levitation technology," IEEE/ASME Trans. Mechatronics, vol. 9, no. 2, pp. 384-391, Jun. 2004.

[9] S. Devasia, E. Eleftheriou, and S. O. R. Moheimani, "A survey of control issues in nanopositioning," IEEE Trans. Contr. Syst. Technol., vol. 15 , no. 4, pp. 689-703, Jul. 2007.

[10] A. J. Fleming, S. S. Aphale, and S. O. R. Moheimani, "A new method for robust damping and tracking control of scanning probe microscope positioning stages," IEEE Trans. Nanotechnol., vol. 9, no. 4, pp. 438-448, Jul. 2010.

[11] A. Sebastian, A. Pantazi, R. Moheimani, H. Pozidis, and E. Eleftheriou, "Achieving sub-nanometer precision in a MEMS storage device during self-servo write process," IEEE Trans. Nanotechnol., vol. 7, no. 5, pp. 586595, Sep. 2008.

[12] S. O. R. Moheimani, "A survey of recent innovations in vibration damping and control using shunted piezoelectric transducers," IEEE Trans. Contr. Syst. Technol., vol. 11, no. 4, pp. 482-494, Jul. 2003.

[13] J. L. Fanson and T. K. Caughey, "Positive position feedback control for large space structures," AIAA J., vol. 28, no. 4, pp. 717-724, 1990.

[14] B. Bhikkaji, M. Ratnam, A. J. Fleming, and S. O. R. Moheimani, "Highperformance control of piezoelectric tube scanners," IEEE Trans. Contr. Syst. Technol., vol. 15, no. 5, pp. 853-866, Sep. 2007.

[15] H. R. Pota, S. O. R. Moheimani, and M. Smith, "Resonant controllers for smart structures," Smart Mater. Struct., vol. 11, pp. 1-8, 2002.
[16] S. Salapaka, A. Sebastian, J. P. Clevland, and M. V. Salapaka, "High bandwidth nano-positioner: A robust control approach," Rev. Sci. Instrum., vol. 73, no. 9, pp. 3232-3241, Sep. 2002.

[17] S. S. Aphale, A. J. Fleming, and S. O. R. Moheimani, "Integral resonant control of collocated smart structures," Smart Mater. Struct., vol. 16, pp. 439-446, Feb. 2007.

[18] E. Pereira, S. S. Aphale, V. Feliu, and S. O. R. Moheimani, "Integral resonant control for vibration damping and precise tip-positioning of a singlelink flexible manipulator," IEEE/ASME Trans. Mechatronics, vol. 16, no. 2, pp. 232-240, Apr. 2011

[19] Y. K. Yong, S. S. Aphale, and S. O. R. Moheimani, "Design, identification, and control of a flexure-based XY stage for fast nanoscale positioning," IEEE/ASME Trans. Mechatronics, vol. 8, no. 1, pp. 46-54, Jan. 2009.

[20] B. Basu and S. R. K. Nielsen, "A multi-modal control using a hybrid pole-placement integral resonant controller (PPIR) with experimental investigations," Struct. Contr. Health Monitor., vol. 18, pp. 191-206, 2011.

[21] I. M. Diaz, E. Pereira, and P. Reynolds, "Integral resonant control scheme for cancelling human-induced vibrations in light-weight pedestrian structures," Struct. Contr. Health Monitor., vol. 19, no. 1, pp. 55-69, 2011.

[22] I. R. Peterson and A. Lanzon, "Feedback control of negative-imaginary systems," IEEE Contr. Syst. Mag., vol. 30, no. 5, pp. 54-72, Oct. 2010.

[23] B. Bhikkaji, S. O. R. Moheimani, and I. R. Petersen, "A negative imaginary approach to modeling and control of a collocated structure," IEEE/ASME Trans. Mechatronics, vol. 17, no. 4, pp. 717-727, Aug. 2012.

[24] E. Eleftheriou and S. O. R. Moheimani, Control Technologies for Emerg ing Micro and Nanoscale System, (Lecture Notes in Control and Information Sciences), vol. 413. New York, NY, USA: Springer-Verlag, 2011.

[25] B. Bhushan, Springer Handbook of Nanotechnology, 3rd ed. New York, NY, USA: Springer-Verlag, 2010.

[26] S. Devasia, E. Eleftheriou, and S. O. R. Moheimani, "A survey of control issues in nanopositioning," IEEE Trans. Contr. Syst. Technol., vol. 15, no. 5 , pp. 802-823, Sep. 2007.

[27] S. O. R. Moheimani. (2008, Jul.). Invited review article: Accurate and fast nanopositioning with piezoelectric tube scanners: Emerging trends and future challenges. Rev. Sci. Instrum., [Online]. 79(7), article number: 071101. Available:http://mechatronics.newcastle.edu.au/lab/ PDF/images/J08j.jpg

[28] R. L. Clark, "Accounting for out-of-bandwidth modes in the assumed modes approach: Implications on collocated output feedback control," Trans. ASME J. Dyn. Syst. Meas. Control, vol. 119, pp. 390-395, 1997.

[29] G. C. Goodwin, S. F. Graebe, and M. Salgado, Control System Design. Englewood Cliffs, NJ, USA: Prentice-Hall, 2000.

[30] A. Preumont, B. de Marneffe, A. Deraemaeker, and F. Bossens, "The damping of a truss structure with a piezoelectric transducer," Comput. Struct., vol. 86, pp. 227-239, 2008.

[31] S. S. Aphale, B. Bhikkaji, and S. O. R. Moheimani, "Minimizing scanning errors in piezoelectric stack-actuated nanopositioning platforms," IEEE Trans. Nanotechnol., vol. 7, no. 1, pp. 79-90, Jan. 2008.

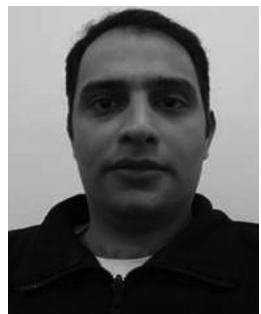

Mohammad Namavar (S'11) received the B.Sc. degree in electrical/control engineering from the Amirkabir University of Technology, Tehran, Iran, in 2004. Since 2010, he has been working toward the Ph.D. degree at the University of Aberdeen, Aberdeen, U.K.

He worked in the oil industry for six years before starting his Ph.D. degree. His current research interests include vibration control, nanopositioning, mathematical system modeling, and process control.

Mr. Namavar is funded by the College of Physical Sciences Research Studentship. In 2012, he was the recipient of the Principal's Excellence Fund, University of Aberdeen. 


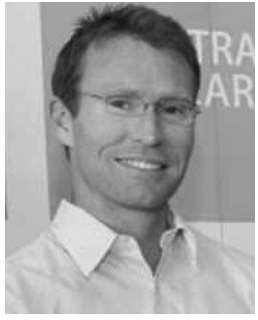

Andrew J. Fleming (M'00) received the B.E.E. and Ph.D. degrees from The University of Newcastle, Callaghan, Newcastle, Australia, in 2000 and 2004, respectively.

He is currently a Senior Lecturer and Australian Research Council Fellow in the School of Electrical Engineering and Computer Science, The University of Newcastle. He is the coauthor of two books, several patent applications, and more than 100 journal and conference papers. His research interests include nanopositioning, high-speed scanning probe microscopy, nanofabrication, and microcantilever sensors.

Dr. Fleming received the Newcastle Innovation Rising Star Award, the IEEE TRAnSACtions on CONTROL Systems TeChnOlogy Outstanding Paper Award, The Australian Control Conference Best Student Paper Award, The University of Newcastle Researcher of the Year Award, and the Faculty of Engineering and Built Environment Award for Research Excellence.

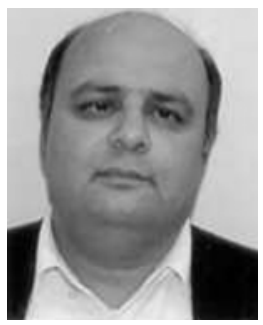

Majid Aleyaasin was born in 1959. He received the B.Eng. degree from Mashhad University, Mashhad, Iran, in 1980, the M.Sc. degree from Sharif University, Tehran, Iran, in 1986, and the Ph.D. degree in mechanical engineering from the University of Bradford, Bradford, U.K., in 2001.

He worked in power generation industries up to 1984. He was a Lecturer of mechanical engineering at Mashhad University until 1997 and was a Research Fellow at UMIST and the University of Manchester until December 2005. Since 2006, he has been a Lecturer in the School of Engineering, University of Aberdeen, Aberdeen, U.K. His research interests include the broad field of applied dynamics of solids and structure.
K. Nakkeeran (M'05) received the B.Eng. degree from Coimbatore Institute of Technology, Coimbatore, India, in 1993, and the M.Tech. and Ph.D. degrees from Anna University, Chennai, India, in 1995 and 1998, respectively.

In 1999, he joined the Institute of Mathematical Sciences, Chennai, where he was a Postdoctoral Fellow for ten months. In 1999, he was a Research Associate in the Department of Physics, University of Burgundy, Dijon, France. In 2002 , he was a Postdoctoral Fellow in the Department of Electronic and Information Engineering, Hong Kong Polytechnic University, Kowloon, Hong Kong. Since 2005, he has been a Lecturer in the School of Engineering, University of Aberdeen, Aberdeen, U.K. His current research interests include solitons, fiber lasers, modeling and simulations of optical devices, long-haul optical fiber communications, and nonlinear science.

Dr. Nakkeeran is a member of the Optical Society of America and the Institution of Engineering and Technology.

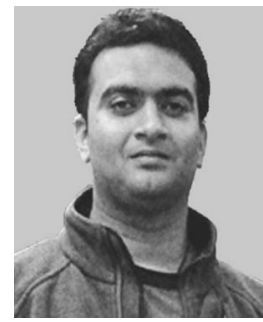

Sumeet S. Aphale (M'09) received the Bachelor's degree from the University of Pune, Pune, India, in 1999, and the M.S. and Ph.D. degrees both from the University of Wyoming, Laramie, WY, USA, in 2003 and 2005, respectively, all in electrical engineering.

In June 2009, he joined the New Blood Lectureship position in the School of Engineering, University of Aberdeen, Aberdeen, U.K. His research interests include robot kinematics and control, vibration control applications, as well as design and control of nanopositioning systems for applications such as scanning probe microscopy. He was a member of the Australian Research Council's Centre of Excellence for Complex Dynamic Systems and Control housed at The University of Newcastle, Australia, from 2005 to 2008 and later moved to the University of Aberdeen as a Research Fellow with the Centre for Applied Dynamics Research. 